\title{
A importância da gestão correta da condição crônica na Atenção Primária à Saúde para o enfrentamento do Covid-19 em Uberlândia, Minas Gerais
}

\author{
Rubia Pereira Barra, Edgar Nunes de Moraes, André Augusto Jardim, Karina Kelly de Oliveira, \\ Poliana Castro de Resende Bonati, Ana Cristina Issa, Carla Jorge Machado
}

\section{RESUMO}

A pandemia pela COVID-19 trouxe a necessidade de novas estratégias para o seu enfrentamento pela atenção primária à saúde, uma vez que abordagens convencionais poderão não ser efetivas. A infecção pelo SARS-Cov-2 tem como principais alvos os idosos e os indivíduos com hipertensão arterial, diabetes mellitus e doença cardiovascular prévia. No município de Uberlândia-MG, este grupo populacional, elemento central da gestão da saúde, vem sendo estratificado por risco, conforme propõe modelo de atenção as condições crônicas. A estratificação de risco tem sido fundamental para o enfrentamento da crise da COVID-19, sendo planejada não apenas de acordo com a idade das pessoas idosas, e sim a partir do índice de vulnerabilidade clínico-funcional, aliando a isto a estratificação de risco de hipertensão e diabetes daqueles idosos portadores destas condições crônicas.

Palavras-chave: Planificação da Atenção à Saúde; Atenção Primária à Saúde; Condições Crônicas; Saúde do Idoso.

\section{ABSTRACT}

The COVID-19 pandemic brought about the need for new strategies for coping with primary health care, since conventional approaches may not be effective. SARS-Cov-2 infection targets the elderly and individuals with hipertension, diabetes and previous cardiovascular disease. In the city of Uberlândia, State of Minas Gerais, Brazil, this population group, a central focus of the health management, has been stratified by risk, as proposed by the care model for chronic conditions. Risk stratification was fundamental for coping with the COVID-19 crisis, being planned not only according to the age of the elderly, but also on the clinical functional vulnerability index, adding to this the risk stratification of hypertension and diabetes of those elderly people with chronic conditions.

Keywords: Health Care Planning; Primary Health Care; Chronic Conditions; Health of the Elderly.
Revista da Rede APS 2020

Publicada em: 15/04/2020

DOI:10.14295/aps.v2i1.64

Rubia Pereira Barra

(Centro Colaborador da Planificação da Atenção em Saúde, Uberlândia, Minas Gerais, Brasil);

Edgar Nunes de Moraes Ana Cristina Issa Carla Jorge Machado (Universidade Federal de Minas Gerais, Belo Horizonte, Minas Gerais, Brasil);

André Augusto Jardim Karina Kelly de Oliveira Poliana Castro de Resende Bonati

(Secretaria Municipal de Saúde de Uberlândia, Minas Gerais, Brasil)

Correspondência para: Rubia Pereira Barra rpbarra@hotmail.com 

C.J.

\section{INTRODUÇÃO}

A pandemia pela COVID-19 evidencia uma oportunidade para avaliação da organização dos sistemas de saúde em todo mundo. Diante desse cenário, o município de Uberlândia apresenta sua experiência no remodelamento da Rede de Atenção à Saúde (RAS), em parceria com o Conselho Nacional de Secretários de Saúde (CONASS). O município atua como Centro Colaborador da Planificação da Atenção à Saúde (PAS) (GUIMARÃES; CAVALCANTI; LINS, 2018) e, desde 2017, desenvolve o processo de organização das RAS sob a perspectiva da coordenação da Atenção Primária à Saúde (APS), que no município recebeu o nome Qualifica SaUDI, direcionando a atenção à população com condições crônicas de saúde, sobretudo para a pessoa idosa e para os indivíduos portadores de hipertensão arterial e diabetes mellitus.

A PAS usa como referencial teórico a Construção Social da Atenção Primária em Saúde (MENDES et al, 2019) e o Modelo de Atenção as Condições Crônicas (MENDES, 2012), cuja implementação exige mudanças na forma como a atenção à saúde é prestada. O modelo propõe que haja estratificação do risco das pessoas com condições crônicas. A diferenciação por risco dos usuários leva em consideração não apenas características individuais, mas também tipos e lugares de atenção e sua concentração relativa em cada grupo populacional. Dessa forma, pessoas com condições crônicas e com menores riscos são orientadas por profissionais de saúde especialmente com base em tecnologias de auto-cuidado apoiado e com foco na APS, enquanto aqueles com condições de alto e muito alto riscos necessitam de atenção profissional especializada, com maior volume de cuidados realizado pela equipe de saúde, e sempre com a coparticipação da APS.

A estratificação de risco dos usuários, que teve seu início em Uberlândia em 2018, utilizou como estratégia:

-População idosa: o instrumento utilizado foi o Índice de Vulnerabilidade Clínico Funcional (IVCF-20) desenvolvido a partir do modelo multidimensional de saúde do idoso, com o objetivo de rastrear a fragilidade (avaliação de risco) e permitir o manejo de condições crônicas de saúde frequentes na pessoa idosa, que são pouco reconhecidas no atendimento tradicional do idoso, como declínio funcional, quedas, polifarmácia, alterações cognitiva e do humor, incontinência urinária, dentre outras (BRASIL, 2019). Idosos com pontuação entre 0 a 6 pontos são considerados robustos; entre 7 a 14 pontos são considerados pré-frágeis e com 15 pontos ou mais são considerados frágeis. A presença de IVCF $\geq 15$ pontos é um marcador da presença de declínio funcional e incapacidades, associados a maior risco de desfechos adversos, como maior internação e óbito. Tais idosos são encaminhados para a atenção ambulatorial especializada em saúde do idoso, restabelecendo a coerência entre demanda e oferta, otimizando a utilização dos recursos municipais (MORAES, et al, 2016).

- População hipertensa e diabética: a estratégia de estratificação de risco utilizou instrumentos tradicionais para avaliação da gravidade, como medida da pressão arterial, avaliação do risco cardiovascular, presença de lesão de órgãosalvo e avaliação da hemoglobina glicada. O instrumento utilizado foi o descrito na Nota Técnica "Normatização da Estratificação de Risco para Diabetes Mellitus e Hipertensão Arterial", da SMS de Uberlândia-MG (UBERLÂNDIA, 2019), baseada nas Diretrizes das respectivas Sociedades de Especialidades.

O panorama do município em termos de estratificação dos idosos encontra-se na Tabela 1. A população de indivíduos com 60 anos ou mais, cadastrados no Sistema Municipal de Saúde na APS é de cerca de 80.000 habitantes, dos quais 35.782 mil idosos estão inscritos nos programas de atenção à saúde do idoso, hipertensão e diabetes, com a seguinte distribuição: idosos frágeis (13,3\%), idosos em risco de fragilização $(20,1 \%)$ e idosos robustos (66,6\%). Entre os idosos robustos, a proporção de portadores de comorbidades é menor, se comparada aos demais grupos 
Barra, R. P.; Moraes, E. N.; Jardim, A. A.; Oliveira, K. K.; Bonati, P. C. R.; Issa, A. C.; Machado, C.J.

Tabela 1 - Prevalências de HAS e diabetes mellitus nos idosos cadastrados no Município do Uberlândia por grupos selecionados, dez 2018 a fev 2020

\begin{tabular}{lccccc}
\hline Estrato de Risco & Total & Hipertensos & Diabéticos & $\begin{array}{c}\text { Sem } \\
\text { comorbidade }\end{array}$ & $\begin{array}{c}\text { Diabéticos } \\
\text { e } \\
\text { Hipertenso } \\
\text { s }\end{array}$ \\
& $\mathrm{N}(\%)^{*}$ & $\mathrm{~N}(\%)$ & $\mathrm{N}(\%)$ & $\mathrm{N}(\%)$ & $\mathrm{N}(\%)$ \\
\hline Frágil & 4.743 & 3.250 & 1.582 & 1.259 & 1.348 \\
& $(13,3)$ & $(68,5)$ & $(33,4)$ & $(26,5)$ & $(28,4)$ \\
Pré-Frágil & 7.196 & 4.939 & 2.229 & 1.919 & 1.891 \\
& $(20,1)$ & $(68,6)$ & $(30,9)$ & $(26,7)$ & $(26,2)$ \\
Robusto & 23.843 & 13033 & 4741 & 9.766 & 3.697 \\
& $(66,6)$ & $(54,6)$ & $(19,8)$ & $(41,0)$ & $(15,5)$ \\
Total & 35.782 & 21.222 & 8.552 & 12.944 & 6.936 \\
& $(100,0)$ & $(59,3)$ & $(23,9)$ & $(36,1)$ & $(19,4)$ \\
\hline
\end{tabular}

Fonte: NIT/SMS Uberlândia-MG. Notas: *foi calculada a distribuição percentual dos estratos na população total atendida, e não as prevalências; houve diferenças significativas entre todas as prevalências $(p<0,05)$, exceto: estratos frágil e pré-frágil no grupo de hipertensos $(p=0,896)$; frágil e pré-frágil no grupo sem comorbidade $(p=0,882)$; o teste estatístico utilizado foi a comparação de proporções para grandes amostras (software Stata/SE for Mac 12.0)

Quase dois terços dos idosos de Uberlândia apresenta alguma comorbidade ( $n=22.838$; $63,9 \%)$, como hipertensão arterial ou diabetes mellitus, de forma isolada ou associada (comorbidade). A prevalência geral encontrada de hipertensão arterial sistêmica (HAS) e diabetes mellitus foi de, respectivamente, $59 \%$ e $23,9 \%$. A HAS $(68,5 \%)$ e o diabetes mellitus $(33,4 \%)$ foram mais prevalentes no idoso frágil, semelhante ao que foi observado no idoso préfrágil. Cerca de $19,4 \%$ da população idosa apresentava uma associação entre HAS e diabetes mellitus, que foi significativamente mais prevalente no idoso frágil $(28,4 \%)$, quando comparado com o idoso pré-frágil $(26,3 \%)$ e robusto $(15,5 \%)(p<0,05)$.

Do total dos 3.484 idosos frágeis com hipertensão arterial e diabetes mellitus, 979
(28\%) foram estratificados como de muito alto risco; 1.251 (36\%) como risco alto; 1107 (32\%); como risco médio e 147 (4\%) como risco baixo, conforme critérios estabelecidos pela nota técnica adotada.

Observa-se uma alta prevalência de hipertensão arterial e diabetes mellitus na população idosa. Isto reforça a hipótese da causalidade cumulativa e sinérgica (Figura 1), na qual a maior taxa de mortalidade da COVID-19 observada no idoso, deve-se, sobretudo, à associação entre hipertensão arterial e diabetes mellitus, que são frequentes no idoso (BRASIL, 2020). Além disso, deve-se considerar o maior risco de declínio funcional e incapacidades neste grupo populacional, que também aumenta significativamente o risco de desfechos adversos diante de qualquer condição aguda de saúde. 

C.J.

Dessa forma, a maior mortalidade seria multifatorial, não só como consequência da idade e da presença de hipertensão arterial e/ou diabetes mellitus, mas, também, da maior vulnerabilidade clínico-funcional presente nos idosos com dependência funcional e incapacidades. Tais fatores de risco não são mutuamente exclusivos e podem apresentar caráter cumulativo e sinérgico.

Figura 1 - Hipótese da causalidade cumulativa e sinérgica na COVID-19

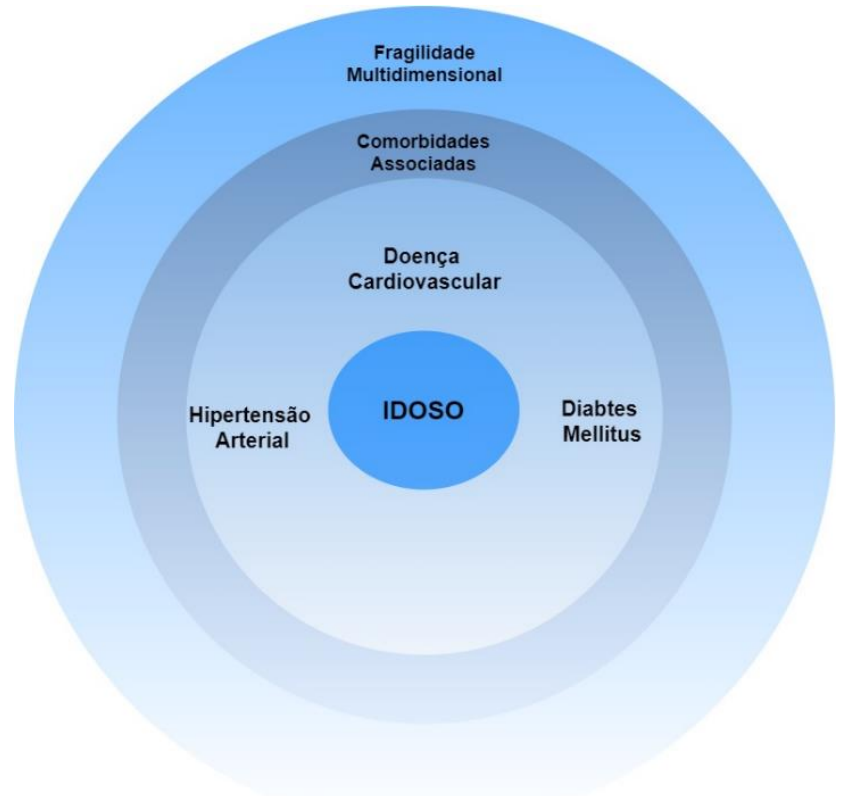

Fonte: elaborado pelos autores.

Assim, o enfrentamento da crise da COVID-19 em Uberlândia foi planejado não apenas de acordo com a idade das pessoas idosas, mas a partir do índice de vulnerabilidade clínico funcional, aliando-se a isto a estratificação de risco de hipertensão e diabetes daqueles idosos portadores destas condições crônicas.

\section{AÇÕES PARA O ENFRENTAMENTO DA} COVID-19 PELA ATENÇÃo PrimÁria À

\section{SAÚDE}

O risco de complicações pela COVID-19 é maior nas pessoas idosas dependentes com comorbidades múltiplas, particularmente nos idosos frágeis, e devem ser monitorados rigorosamente pela atenção primária (BRASIL, 2020). A partir daí, foi elaborado material específico para o momento, por meio de vídeos- aulas, fluxogramas, matrizes de gerenciamento e planos de ações. Todas as ações foram planejadas de forma a evitar que as pessoas idosas se mantivessem em isolamento/distanciamento social (MORAES, 2020), conforme as orientações do Ministério da Saúde, utilizando-se as seguintes estratégias:

- Cada equipe de atenção primária organizou planilha de registro coletivo, a partir do IVCF-20, cruzando os dados com a estratificação de risco de hipertensão e diabetes, iniciando-se a relação pelas pessoas de maior risco;

- A campanha de influenza está acontecendo nos domicílios, levando em consideração a estratificação de risco, iniciando pelos idosos frágeis, de muito alto e alto risco para hipertensão e diabetes, de forma a vacinar todas as pessoas do território das Unidades Básicas de 

C.J.

Saúde (UBS). São também realizadas as orientações de medidas protetivas e de educação em saúde;

- Foi disponibilizado o telefone da UBS para qualquer necessidade, chamando a atenção para que os idosos e familiares evitem sair de suas residências para se dirigirem à unidade de saúde;

- Utilização de todos os profissionais disponíveis para a realização das visitas domiciliares de forma que todos os idosos, cuidadores e familiares, da área de abrangência recebam as orientações em um menor espaço de tempo;

- Na presença de suspeita da infecção pelo coronavírus, as famílias estão sendo orientadas a fazer contado com a UBS antes de levar o idoso frágil para atendimento de urgência (pronto socorro, UPAs, etc), onde o risco de contaminação é elevado e o acolhimento do paciente será conturbado neste momento;

-Estão sendo realizadas visitas nas ILPI do território, com a aplicado da vacina antiinfluenza e orientações de medidas protetivas;

- O acompanhamento das pessoas idosas e com comorbidades está sendo realizado por meio de vistas domiciliares, contato telefônico ou WhatsApp, visto que estas pessoas já possuem suas próximas consultas programadas e agendadas segundo a estratificação de risco e parametrização, seguindo os passos abaixo:

-Verificação semanal das consultas programadas;

-Identificação das pessoas usuárias de acordo com a estratificação de risco;

- Pessoas estratificadas como muito alto risco devem ter sua consulta realizada no domicilio pelo médico ou enfermeiro, de acordo com a parametrização, atualizando o plano de cuidados;

- Pessoas estratificadas como alto risco devem ser atendidas por teleconsulta, realizada por telefone ou WhatsApp pelo médico (CONSELHO FEDERAL DE MEDICINA, 2018) ou enfermeiro
(CONSELHO FEDERAL DE ENFERMAGEM, 2020), atualizando o plano de cuidados, avaliando a necessidade de visita domiciliar posterior;

- Pessoas estratificadas como médio e baixo risco recebem contato telefônico ou WhatsApp qualificado (verificando alterações inapropriadas da glicemia capilar; níveis pressóricos), pela equipe multidisciplinar, sempre consultando a história clínica do paciente e verificando os relatos de situações que fujam da normalidade. Em caso de relato de anormalidade, a enfermeira da unidade deve ser avisada para que possa agendar consulta domiciliar ou teleconsulta.

O fato de trabalhar com a modelo atenção às condições crônicas foi fundamental para o enfrentamento da COVID-19:

"Estou aqui novamente assistindo esse vídeo, e percebendo o quanto a estratificação de risco é importante. Nesse caso do idoso, por exemplo nos permite tomar decisões importantes de conduta, se caso for preciso num momento de pandemia, ou crises como essa que estamos vivendo." (relato de Mariana Machado dos Santos Pereira, enfermeira da UBSF Santa Luzia).

\section{REFERÊNCIAS BIBLIOGRÁFICAS}

BRASIL. Ministério da Saúde. Protocolo de Manejo Clínico do Corona Vírus (COVID-19) na Atenção Primária à Saúde. Brasília: Secretaria de Atenção Primária à Saúde, 2020. 33 p.:il. Disponível em: https://saude.gov.br/images/pdf/2020/marco/3 0/20200330-ProtocoloManejo-ver06-Final.pdf.

Acesso 08 abr 2020.

BRASIL. Ministério da Saúde. Nota Técnica para Organização da Rede de Atenção à Saúde do Foco na Atenção Primária à Saúde e na Atenção Ambulatorial Especializada: SAÚDE DA PESSOA IDOSA (2019). Sociedade Beneficente Israelita Brasileira Albert Einstein. São Paulo: Hospital Israelita Albert Einstein: Ministério da Saúde, 2019.56 p.:il. Disponível em: 

C.J.

https://atencaobasica.saude.rs.gov.br/upload/a rquivos/202001/03091212-nt-saude-do-idosoplanificasus.pdf. Acesso em: 07 abr 2020.

CONSELHO FEDERAL DE ENFERMAGEM. Resolução COFEN № 634/2020 - Autoriza e normatiza, "ad referendum" do Plenário do Cofen, a teleconsulta de enfermagem como forma de combate à pandemia provocada pelo novo coronavírus (Sars-Cov-2), mediante consultas, esclarecimentos, encaminhamentos e orientações com uso de meios tecnológicos, e dá outras providências.

CONSELHO FEDERAL DE MEDICINA. Resolução CFM no 2.227/2018 - Define e disciplina a telemedicina como forma de prestação de serviços médicos mediados por tecnologias.

GUIMARÃES, A. M. D. N.; CAVALCANTI, C. B. C; LINS, Z. S. (Org). Planificação da atenção primária à saúde: um instrumento de gestão e organização da atenção primária e da atenção ambulatorial especializada nas redes de atenção à saúde. CONASS Documenta, v.31. Brasília: CONASS, 2018. 300p. Disponível em: http://www.conass.org.br/consensus/planificac ao-da-atencao-saude-uma-proposta-de-gestaoe-organizacao-da-atencao-primaria-saude-e-daatencao-ambulatorial-especializada-nas-redesde-atencao-saude/. Acesso em: 07 abr 2020.

MENDES, E. V. O cuidado das condições crônicas na atenção primária à saúde: o imperativo da consolidação da estratégia da saúde da família. Brasília: Organização Pan-Americana da Saúde, 2012. 512 p. Disponível em: https://bvsms.saude.gov.br/bvs/publicacoes/cui dado_condicoes_atencao_primaria_saude.pdf. Acesso em: 07 abr 2020.

MENDES, E. V; MATOS M. A. B.; EVANGESLISTA, M. J.; BARRA, R.P. A construção social da atenção primária à saúde. 2 ed. Brasília: Conselho Nacional de Secretários de Saúde - CONASS, 2019. 192 p. Disponível em: https://www.conass.org.br/biblioteca/pdf/ACONSTR-SOC-ATEN-PRIM-SAUDE.pdf. Acesso em: 07 abr 2020.
MORAES, E. N.; CARMO, J.A.; LANNA, F. M.; AZEVEDO R.S.; MACHADO, C.J.; ROMERO D. E. M. Índice de Vulnerabilidade Clínico Funcional-20 (IVCF-20): reconhecimento rápido do idoso frágil. Rev Saude Publica. 2016; v. 50:81. Disponível em:

http://www.scielo.br/scielo.php?pid=S003489102016000100254\&script=sci_arttext\&tlng=p t. Acesso em: 07 abr 2020.

MORAES, E. N. A pandemia por coronavirus e o idoso. Belo Horizonte: Universidade Federal de Minas Gerais. Núcleo de Geriatria e Gerontologia da UFMG. Serviço de Geriatria do HC-UFMG. 2020. mimeo.

UBERLÂNDIA. Secretaria Municipal da Saúde. Nota Técnica Normatização da Estratificação de Risco e Parametrização das Consultas de Atenção à Saúde: Diabetes Mellitus, Hipertensão Arterial, Criança, Gestante e Mulher (2019). Uberlândia: Secretaria Municipal da Saúde, 2019. Mimeo. 